\title{
Utilizzo di Etest nell'antibiogramma diretto da emocoltura su bacilli Gram negativi a crescita rapida e Staphylococcus spp.
}

\author{
Pietro Casella', Giovanna Confalonieri', Donato Longo', Stefano Muttini²
}

' A.O. “Ospedale Civile” di Vimercate: Presidio di Vimercate, Laboratorio analisi chimico cliniche e microbiologiche,

${ }^{2}$ Divisione di terapia intensiva e rianimazione

Etest in direct testing susceptibilities from blood in gram negative rapid growers strains and Staphylococcus spp.

Key words: Etest, Direct susceptibility testing, Blood coltures

\section{SUMMARY}

The objective of this study was to compare $E$ test antibiotic gradient performed directly from positive blood coltures with Vitek 2 System for rapid susceptibility testing.A total of 47 strains of Gram negative rapid growers and 37 strains of Staphylococcus spp. ( 10 strains S. aureus and 27 coagulase negative) were investigated. Direct testing of susceptibility to amikacin, imipenem, piperacillin-tazobactam, and ciprofloxacin was evaluated for Gram negative strains, and to oxacillin,ofloxacin, and imipenem for Gram positive. The rate of correlation resulted $99.4 \%$ for Gram negative strains (187//88) and 97\% for Gram positive strains (144//48). Major errors (i.e. reported as resistant by the Etest but shown to be sensible by the Vitek) were observed and regarding one strain of $P$. aeruginosa for imipenem and two strains of Staphylococcus coagulase negative for oxacillin and imipenem. Etest resulted useful for direct testing susceptibility from blood and may lead to cost reducttion.

\section{INTRODUZIONE}

Nonostante la disponibilità di chemioantibiotici efficaci verso buona parte dei batteri isolati da sangue la mortalità tra pazienti con documentata batteriemia è ancora elevata (14). L'importanza clinica dell'emocoltura si è tradotta in una maggior attenzione per quest'indagine sia da parte dei microbiologi che dei curanti (14). Uno di questi aspetti riguarda la tempestività della comunicazione dei risultati, in particolare degli antibiogrammi eseguiti direttamente su coltura positiva. Ciò consente di anticipare in via preliminare l'antibiogramma di 24 ore $(7,8)$.

Scopo del lavoro è stato quello di confrontare un metodo a gradiente di diffusione diretto da emocolture positive (Etest) con l'antibiogramma standardizzato eseguito con Vitek 2 per bacilli Gram negativi, con morfologia microscopica riconducibile ad enterobatteri o Pseudomonas spp, e cocchi Gram positivi, morfologia stafilococchi, con un limitato numero di antibiotici ad elevato impatto terapeutico.

\section{MATERIALI E METODI}

Emocolture: eseguite con strumentazione a monitoraggio continuo BacT Alert, tempo di incubazione 5 giorni. Ogni set comprensivo di flaconi FAN Aerobi + Flaconi FAN Anaerobi.

Campioni: l'indagine è stata condotta su 50 set positivi consecutivi per Gram negativi e 37 Gram positivi morfologia stafilococchi (almeno 2 flaco- ni positivi per lo stesso paziente). Gli isolati sono riferiti a pazienti diversi.

Identificazioni e antibiogrammi definitivi sono stati condotti con Vitek 2 con le seguenti card: IDGPC, ID-GNB, AST-N019, AST-P523 come raccomandato dal produttore.

E test: dopo l'esecuzione della colorazione di Gram, 1-2 ml di emocoltura positiva venivano travasati in una provetta di plastica. La semina è stata fatta con tampone in piastre di Mueller Hinton Agar (MHA) $+5 \%$ di sangue di pecora, e per oxacillina $\mathrm{MHA}+2 \%$ di $\mathrm{NaCl}$.

Antibiotici saggiati: (E test $\mathrm{AB}$ Biodisk) amikacina, imipenem, piperacillina/tazobactam e ciprofloxacina (Gram negativi), oxacillina, ofloxacina, vancomicina e imipenem per stafilococchi. Incubazione: $35^{\circ} \mathrm{C}, \mathrm{O} . \mathrm{N}$. e 24 ore per oxacillina. Il confronto è stato condotto solo per classi di appartenenza (S,I,R) poiché le MIC di Vitek 2 non sono determinate direttamente mentre quelle rilevate con $\mathrm{E}$ test sono dirette. Interpretazione di $\mathrm{E}$ test secondo NCCLS M100 S12 (12). Per la corretta interpretazione della MIC per oxacillina è stato eseguito il test della coagulasi rapida (Dryspot Staphytect Plus: Oxoid) sulle piastre di primo isolamento delle emocolture positive per Staphylococcus spp, poiché le break point di $S$. aureus sono diverse dagli stafilococchi coagulasi negativi (12).

Per gli stafilococchi i dati di Vitek 2 per imipenem sono stati dedotti dalla resistenza alla penicillina e dalla sensibilità all'oxacillina. 


\section{RISULTATI}

I ceppi isolati: 50 Gram negativi e 37 stafilococchi, sono riportati in tabella 1 . Per 3 Gram negativi visti nell'esame microscopico diretto non è stato possibile eseguire il confronto perché 2 erano anaerobi (B. fragilis) e uno S. maltophilia (non eseguibile con Vitek 2 con gli antibiotici considerati).

Gram negativi: i risultati sono riportati in tabella 2. La percentuale totale delle sensibilità per le combinazioni ceppi/antibiotici considerate è stata del $94 \%$ (177/188). Le resistenze rilevate erano le seguenti: 11 per ciprofloxacina (9 E. coli, 2 P. mirabilis, $1 P$. aeruginosa), una per piperacillina/tazobactam e una per imipenem. I risultati concordanti sono stati del $99.4 \%$. Il solo dato discordante è stato rilevato con ceppo di $P$. aeruginosa (resistente per Etest e sensibile per Vitek: major error).

Gram positivi: i risultati sono riportati in tabella 3. La percentuale totale di sensibilità per le combinazioni ceppi/antibiotici considerati è stata del $65.5 \%$ (97/148). Tutti i ceppi considerati erano sensibili alla vancomicina, 18 erano resistenti ad oxacillina (15 CNS e $3 S$. aureus), 18 resistenti a imipenem (15 CNS e $3 S$. aureus) e 14 resistenti a ofloxacina (11 CNS e $3 S$. aureus).

Rispetto alla totalità dei confronti i risultati concordanti sono stati il 97\% (144/148). Le discordanze rilevate si sono verificate in 2 ceppi di CNS. Entrambi erano resistenti per E test con MIC di $0.5 \mu \mathrm{g} / \mathrm{ml}$ (break point: $0.25 \mu \mathrm{g} / \mathrm{ml}$ ) e sensibili con Vitek.

\section{DISCUSSIONE}

Nonostante la disponibilità di antibiotici efficaci verso quasi tutti i batteri isolati da sangue la mortalità in pazienti con documentata batteriemia è ancora elevata (20-50\%) e molte sepsi di natura nosocomiale sono causate da batteri con maggior resistenza agli antibiotici $(1,14)$. L'importanza dell'emocoltura si è tradotta in una maggior attenzione da parte dei microbiologi e delle società di diagnostici portando allo sviluppo di nuovi approcci metodologici e strumentali per ottimizzare l'isolamento di batteri e funghi da sangue (1, 14). Con l'introduzione dei sistemi non invasivi a monitoraggio continuo i tempi di rilevazione dei campioni positivi si sono drasticamente ridotti (14) e la maggior parte delle batteriemie significative sono rilevabili entro le prime $24-48$ ore (14). Ulteriori sforzi sono stati fatti anche per velocizzare la gestione dei campioni positivi segnalando la positività al curante e allestendo identificazioni ed antibiogrammi preliminari direttamente sulla coltura positiva dopo colorazione di $\operatorname{Gram}(2,6)$. Anche se i test diretti di sensibilità sono da considerarsi provvisori e vanno confermati, essi sono in buona parte sostanzialmente sovrapponibili a quelli standardizzati ed il loro impiego è raccomandato data la potenziale gravità della situazione clinica $(4,5,7,8)$. L'esecuzione dell'antibiogramma diretto era diffusa nel 31\% dei laboratori USA già nel $1986(9$, 10), mentre in Italia solo il $17.9 \%$ dei laboratori lo

Tabella I. Ceppi batterici isolati

\begin{tabular}{lccc}
\hline GRAM NEGATIVI & TOTALE 47 & GRAM POSITIVI & TOTALE 37 \\
\hline E. coli & 30 & Stafilococchi coagulasi negativi (CNS) & 27 \\
\hline K. pneumoniae & 2 & Staphylococcus aureus & 10 \\
\hline Proteus spp & 3 & & \\
\hline Cirobacter spP & 2 & & \\
\hline E. cloacae & 1 & & \\
\hline S. marcescens & $\mathrm{I}$ & & \\
\hline Salmonella enterica & $\mathrm{I}$ & & \\
\hline P. aeruginosa & 6 & & \\
\hline Acinetobacter spP & $\mathrm{I}$ & & \\
\hline
\end{tabular}

Tabella 2. Confronto fra Etest e Vitek 2 per batteri Gram negativi

\begin{tabular}{cccccc}
\hline E TEST & VITEK 2 & AMIKACINA & IMIPENEM & PIPERACILLINA/TAZOBACTAM & CIPROFLOXACINA \\
\hline $\mathrm{S}$ & $\mathrm{S}$ & 47 & 46 & 46 & 37 \\
\hline $\mathrm{R}$ & $\mathrm{R}$ & 0 & 0 & $\mathrm{I}$ & 10 \\
\hline $\mathrm{S}$ & $\mathrm{R}$ & 0 & 0 & 0 & 0 \\
\hline $\mathrm{R}$ & $\mathrm{S}$ & 0 & $\mathrm{I}$ & 0 & 0 \\
\hline & & 47 & 47 & 47 & 47 \\
\hline
\end{tabular}

Tabella 3. Confronto fra Etest e Vitek 2 per Staphylococcus spp

\begin{tabular}{cccccc}
\hline ETEST & VITEK 2 & OXACILLINA & VANCOMICINA & OFLOXACINA & IMIPENEM \\
\hline $\mathrm{S}$ & $\mathrm{S}$ & 17 & 37 & 23 & 17 \\
\hline $\mathrm{R}$ & $\mathrm{R}$ & $\mathrm{I}$ & 0 & 14 & 18 \\
\hline $\mathrm{S}$ & $\mathrm{R}$ & 0 & 0 & 0 & 0 \\
\hline $\mathrm{R}$ & $\mathrm{S}$ & 2 & 0 & 0 & 2 \\
\hline & & 37 & 37 & 37 & 37 \\
\hline
\end{tabular}


esegue attualmente (6). Nel nostro laboratorio è in uso il sistema Vitek 2 per la routine ed E test per emofili, pneumococchi e streptococchi viridanti. La scelta di Etest per l'antibiogramma diretto delle emocolture è stata fatta per la flessibilità, la facilità di esecuzione e la possibilità di ottenere le risposte in MIC dirette (11). Con il reparto di terapia intensiva che utilizza un protocollo di "de escalation therapy" per i pazienti con sepsi severa abbiamo concordato l'utilizzo di pochi antibiotici (per contenere i costi di E test) ad ampio impatto terapeutico per Gram negativi e Gram positivi.

La percentuale di concordanza per tutte le combinazioni di ceppo-antibiotico saggiate: $99.4 \%$ per i Gram negativi e 97\% per i Gram positivi è sicuramente buona e si allinea con un'altra esperienza italiana positiva (3). Per i tre casi discordanti: $1 P$. aeruginosa e 2 CNS si trattava di Major error (resistenti E test e sensibili Vitek). Poichè il confronto è stato fatto con Vitek e non con un metodo di riferimento NCCLS e considerando che nei casi discordanti considerati le MIC di E test coincidevano con le break point $(16 \mu \mathrm{g} / \mathrm{ml}$ per $P$. aeruginosa con imipenem e $0.5 \mu \mathrm{g} / \mathrm{ml}$ per CNS con oxacillina) non siamo stai in grado di stabilire la reale classe di appartenenza di questi isolati. Il numero di situazioni cliniche in cui è fondamentale la conoscenza della MIC per un corretto trattamento del paziente è limitato probabilmente alle endocarditi ed osteomieliti (11) ma l'utilizzo di E test rappresenta una garanzia per il laboratorista in quanto ci consente di stabilire la distanza della MIC del ceppo testato dalle break point. Ciò secondo noi è di fondamentale importanza per un antibiogramma preliminare. L'utilizzo di un metodo che permette di rilevare direttamente la MIC ci consente infine di interpretare l'oxacillina sia per $S$. aureus che per gli stafilococchi coagulasi negativi in quanto le break point sono diverse nei due casi (12). Tale approccio non è fattibile per esempio con Oxacillin salt agar screening poiché il test è utilizzabile solo per $S$. aureus in quanto usa una solo concentrazione di oxacillina (12). Nella nostra esperienza c'è probabilmente un bias dovuto alla limitata casistica e rappresentatività delle specie batteriche considerate, tuttavia i dati ottenuti, a nostro giudizio, sono incoraggianti sull'utilità clinica per un precoce approccio terapeutico mirato.

\section{CONCLUSIONI}

Nella nostra esperienza Etest si è dimostrato affidabile e di facile esecuzione nell'antibiogramma diretto da coltura per Gram negativi a crescita rapida e Staphylococcus spp per le combinazioni bettere/antibiotico da noi scelte. Lo svantaggio dovuto ai costi elevati può essere arginato con la scelta di pochi ma significativi antibiotici.
Ulteriori approfondimenti dovrebbero essere condotti su una maggiore casistica per confermare quanto rilevato in questa esperienza pilota.

\section{BIBLIOGRAFIA}

1. Brian CS. Clinical Implications of Blood Positive Cultures. Clin Microbiol Rev 1989; 2: 329-53.

2. Calì AM, Gualdi P, Maffei R, Schinella M. Tra microbiologia tradizionale e tecniche molecolari: le possibilità per un laboratorio medico di ridurre il TAT e migliorare l'outcome nella diagnostica delle infezioni del sangue. Microbiol Medica 2002; 17: 96-7.

3. Callegaro A, Basaglia G, Mucignat G, Pascoli L, Tarabini G, Santini G. Uso di E-Test per il saggio di sensibilità diretto da emoculture. Microbiologia Medica 1995; 10: 100-1.

4. Goglio A, Marchiaro G, Notarnicola MA, Piacentini I, Scagnelli M. L'emocoltura nei laboratori italiani. L'igiene moderna 1990; 93: 143-59.

5. Goglio A, Marchiaro G. Affidabilità dell'antibiogramma diretto da emocolture. BML 1993; 1: 41-8.

6. Goglio A, Nicoletti P. Indagine Nazionale sulle Metodiche per Emocoltura in Italia. Microbiologia Medica 2004;19(1):1-13.

7. Health Protection Agency. Standard operative procedure. Investigation of blood cultures (for organisms other than Mycobacterium species). BSOP 37, Issued by Standards Units, Evaluations and Standards Laboratory, http://www.phls.co.uk/dir/hq/sops/bsoppdf/bsop37i3.1.pdf. Issue date: 02.06.03

8. Isemberg HD. Clinical Microbiology Procedures Handbook. American Society for Microbiology, Washington DC, USA 1992.

9. Kehl KSC. Results of Survey of Blood culture Methods: part I Cl Microbiol Newsletter 1986; 8, 117-20.

10. Kehl KSC. Results of Survey of Blood culture Methods: part II Cl Microbiol Newsletter 1986; 8: 127-32.

11. Murray PR, Baron EJ, Jorgensen JH, Pfaller MA, Yolken RH. Manual of Clinical Microbiology, $8^{\text {th }}$ ed, American Society for Microbiology, Washington DC, USA 2003.

12. National Committee for Clincal Laboratory Standards. Performances Standards for Antimicrobial Susceptibility testing; Twelf Informational Supplements M 100-S12, 2002 NCCLS Villanova, $\mathrm{Pa}$.

13. O’Hara CM, Weinstein MP, Miller JM. Manual and Automated Systems for Detection and Identification of Microrganisms. In: Murray PR, Baron EJ, Jorgensen JH, Pfaller MA, Yolken RH. Manual of Clinical Microbiology, $8^{\text {th }}$ ed, American Society for Microbiology, Washington DC, USA.

14. Reimer LG, Wilson ML, Weinstein MP. Update on detection of bacteremia and fungemia. Clin Microbiol Rev 1997; 10: 444-65.

\section{Pietro Casella}

A.O. "Ospedale Civile" di Vimercate Presidio di Vimercate, Laboratorio analisi chimico cliniche e microbiologiche Via C. Battisti 23, - 20059 Vimercate - MI Tel.: 039 6654591; Fax: 03966544772 E-mail: casella.pietro@aovimercate.org 